\title{
NOTES
}

\section{THIN-OXIDE THICKNESS MEASUREMENT IN ELLIPSOMETRY BY A WAFER ROTATION METHOD}

\author{
(Received 19 January 1990; in revised form 10 April 1990)
}

\section{INTRODUCTION}

Ellipsometry is important in the measurement of oxide thickness. Unfortunately, when the oxide thickness is smaller than $200 \AA$, the accuracy of the measured thickness becomes poor since the errors due to the uncertainty of incident angle, $\phi_{0}$, and refractive index, $N$, are significant $[1,2]$. In order to improve this accuracy, several methods have been reported[3-5]. Generally, they are developed without losing the simplicity of the operation of the ellipsometer.

In this work, we propose a simple way to reliably find the thickness of a thin oxide by a wafer rotation method. When one is measuring a thick oxide, there are two fitting parameters, i.e. $T$ and $N$. In such a case, the real deviation of $\phi_{0}$ from $70^{\circ}$ is not important to the accuracy of $T$. Thus optimal $T$ and $N$ are easily found. However, when the thickness is smaller than $200 \AA$, the effect of $\phi_{0}$ is not negligible. Here there are three parameters, i.e. $T, N$ and $\phi_{0}$, need to be determined. This is of course impossible to be optimally solved from two equations[6]. Further information from the sample under test should be given. It is important to note that, since the sensitivity of the deviation of $\phi_{0}$ to $T$ is significant, one should not set a constant $\phi_{0}$ during each repeated measurement. Also, $T$ and $N$ can also vary from each other if the test points are not at the same place on the wafer. This is due to the possible occurrence of oxide thickness nonuniformity during thin oxide growth.

\section{WAFER ROTATION METHOD}

In order to fix the $T$ and $N$, the test point for each repeated measurement should be the same. Figure 1 shows the schematic diagram of our wafer rotation system. The wafer is placed on a rotation stage. The rotation stage is suitably adjusted so that its rotation center is coincident with the incident beam. By rotating the wafer gradually from $0^{\circ}$ to $360^{\circ}$, one can obtain a series of ellipsometric data, i.e. $\psi$ and $\Delta$, based on test points which are located around the same place. It is noted that $\tan \psi$ is the relative amplitude attenuation and $\Delta$ is the relative phase change of the light polarized parallel and perpendicular to the plane of incidence.

To start with, the conventional ellipsometric measurements are carried out. Figure 2(a) shows the fitted data of $T$ and $N$ vs rotation angle $\theta$ under $\phi_{0}=70^{\circ}$. The $\theta$ is increased from $0^{\circ}$ to $360^{\circ}$ in a step of $10^{\circ}$ gradually. It is clear that the $T$ 's are deviated seriously from each other in the range of $150-180 \AA$. The average thickness $T$ is $164 \AA$ and the root mean square deviation of $T$ 's from $T$, i.e. $\Delta T=\left[\Sigma(T-T)^{2} / 36\right]^{1 / 2}$, is $6.65 \AA$. The sensitivity of $\Delta T$, i.e. $S_{\mathrm{T}}=\Delta T / T=4.06 \%$, is large so that one should not take a single measurement datum as the final result. Similarly, from the $N$ 's one can obtain an average index $N$ of 1.405 . The root mean square deviation of $N$ 's is $\Delta N=0.029$. The sensitivity of $\Delta N$, i.e. $S_{N}=\Delta N / N=2.13 \%$, is smaller than
$S_{\mathrm{T}}$. Let the real refractive index have the value of 1.405 . By fitting $T$ and $\phi_{0}$ for $N=1.405$, based on the ellipsometric data measured in Fig. 2(a), one can then obtain the data shown in Fig. 2(b). It is interesting to note that the newly fitted $T$ 's are nearly constant. The average thickness $T$ is $163 \AA$ and the $\Delta T$ and $S_{\mathrm{T}}$ are $0.71 \AA$ and $0.43 \%$, respectively. As can be seen from this result, the $S_{\mathrm{T}}$ has been much improved. The $T$ 's shown in Fig. 2(b) are much more reliable than those shown in Fig. 2(a). Thus one can determine the final thickness from any refitting $T$ as shown in Fig. 2(b).

\section{DISCUSSION AND CONCLUSION}

It is noted that, although the average of the refitted $T$ 's, i.e. $\bar{T}=163 \AA$, is close to the original $T$, i.e. $164 \AA$, the refitting work is necessary since the deviations of $T$ 's between the two procedures are quite different. Reliable $T$ 's are obtainable only when the $\bar{N}$ is correctly found and the $\phi_{0}$ 's are free. Also, it is important to note that the fitted $\phi_{0}$ 's are randomly deviated from $70^{\circ}$. This is reasonable since $\phi_{0}$ is not a constant in each repeated measurement as mentioned above. As can be seen from this figure, the deviations of $\phi_{0}$ from $70^{\circ}$ are very small. Therefore, a small change of $\phi_{0}$, which occurs in a real case, contributes a significant change of $T$ in thin oxide measurements.

In the wafer rotation method, the determination of $N$ is of the most importance. If $N$ deviates considerably from the real $N$, the average of the fitted $\phi_{0}$ 's will not equal $70^{\circ}$. The effects of the systematic errors of $\psi$ and $\Delta$ can also cause the same phenomenon. Therefore, the examination of the difference between the average of the fitted $\phi_{0}$ 's and $70^{\circ}$ gives one a knowledge of how well the $N$ was chosen

Theoretical calculations of the effect of $\phi_{0}$ on $T$ and $N$ are also presented. Figures 3(a) and (b) show simulated thickness $T^{\prime}$ and refractive index $N^{\prime}$ vs given thickness $T$, with $\phi_{0}$ as a parameter. For a given $T$ and a certain $\phi_{0}$, one can find the ellipsometric data of $\psi$ and $\Delta$ under $N=1.46$. Since $\phi_{0}$ is generally set to $70^{\circ}$ in an ellipsometer, the fitted $T^{\prime}$ and $N^{\prime}$ according to $\psi$ and $\Delta$ found under $\phi_{0}=70^{\circ}$ are different from the given $T$ and 1.46 , respectively. As shown in Fig. 3(a), $T^{\prime}$ deviates considerably from the

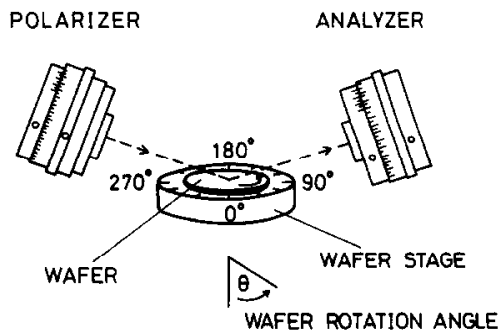

Fig. 1. Schematic diagram of a wafer rotation system. 

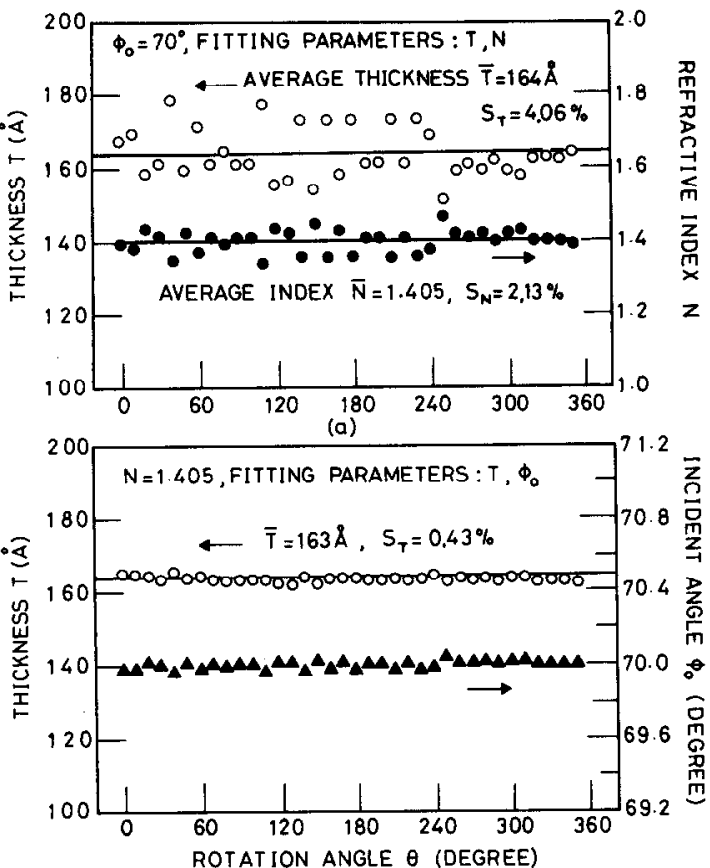

(b)

Fig. 2. (a) Fitted thickness, $T$, and refractive index, $N$, vs wafer rotation angle, $\theta$, under an incident angle, $\phi_{0}$, of $70^{\circ}$. (b) Refitted $T$ and $\phi_{0}$ under $N=1.405$ according to the ellipsometric data measured in (a).

corresponding $T$, as $T$ is less than $200 \AA$. Besides, from Fig. 3(b), the deviation of $N^{\prime}$ from 1.46 increases as $T$ decreases. It is noted that a $\phi_{0}$ less than $70^{\circ}$ implies a $T^{\prime}$ greater than $T$ and a $N^{\prime}$ less than 1.46. This is consistent with the data shown in Figs 2(a) and (b). As shown in Fig. 2(a), an original fitted $T$ greater than $164 \AA$ is accompanied with an original fitted $N$ less than $N(=1.405)$. After refitting $T$ and $\phi_{0}$ under $N=1.405$ as shown in Fig. 2(b), a corresponding $\phi_{0}$ less than $70^{\circ}$ is found. Interestingly, no matter how the original $T$ and $N$ and the fitted $\phi_{0}$ are related, the final refitted $T$ 's are almost the same. Therefore, the wafer rotation method indeed provides an improvement in the thin oxide thickness measurement.

In conclusion, a simple ellipsometric procedure is proposed to find the thin oxide thickness. The procedure involves a rotation of the wafer, to insure that the measured data are obtained from the same point. The improvement shown in this work is significant and is useful in some applications.
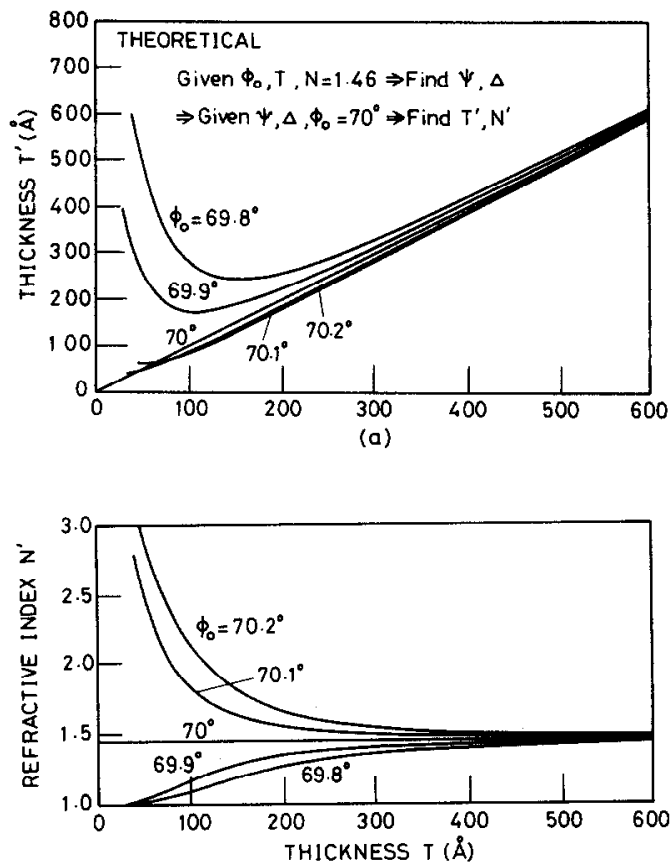

(b)

Fig. 3. Theoretical calculations of (a) simulated thickness, $T^{\prime}$, and (b) refractive index, $N^{\prime}$, vs given thickness, $T$, with incident angle, $\phi_{0}$, as a parameter.

Acknowledgement - The authors want to thank the National Science Council of the Republic of China for the support of this work under Contract No. NSC78-0404-E002-51.

Department of Electrical Engineering National Taiwan University

JENN-Gwo HwU I.-Hsiu Ho

Taipei, Taiwan, R.O.C.

SHOU-PIN CHOU

\section{REFERENCES}

1. J. R. Zeidler, R. B. Kohles and N. M. Bashara, Appl. Opt. 13, 1591 (1974).

2. J. H. Ho, C. L. Lee, C. W. Jen and T. F. Lei, Solid-St. Electron. 30, 973 (1987).

3. J. H. Ho, C. L. Lee and T. F. Lei, Solid-St. Electron. 31, 1321 (1988).

4. F. L. McCrackin, J. opt. Soc. Am. 60, 57 (1970)

5. G. H. Bu-Abbud and N. M. Bashara, Appl. Opt. 20, 2815 (1981).

6. R. J. Archer, J. opt. Soc. Am. 52, 970 (1962). 